\title{
ESTADO DE FRAGILIDAD DE LA SALUD Y TIPO DE INCONTINENCIA URINARIA EN ADULTOS MAYORES VARONES QUE ASISTEN AL CONSULTORIO EXTERNO DE MEDICINA DE UN HOSPITAL PÚBLICO DE II NIVEL ICA, ENERO 2016.
} State of Fragility of Health and Type of Urinary Incontinence In Adults Major Men Who Attend The External Medical Office Of A Public Hospital Of II Level Ica, January 2016.

\section{ARTÍCULO ORIGINAL}

Olinda Oscco - Torres ${ }^{1, a}$.

1. Universidad Privada San Juan Bautista - Filial Ica. Ica, Perú a. Licenciada en Enfermería.

\section{Correspondencia:}

Olinda Oscco - Torres

Dirección: Av Cutervo SN - Ica

Correo electrónico:

olita_4@hotmail.com

Celular: 995020980

Conflicto De Intereses: No declarados.

Financiamiento: Autofinanciado.

Recibido: 25-02-2017

Aceptado: 27-02-2017

Publicado: 19-03-2017

\section{CITAR COMO:}

Oscco-Torres O. Estado de fragilidad de la salud y tipo de incontinencia urinaria en adultos mayores varones que asisten al consultorio externo de medicina de un hospital público de II nivel Ica, Enero 2016. Rev méd panacea. 2017; 6(1): 40-43.

\section{RESUMEN}

Objetivos: Determinar el estado de fragilidad de la salud y tipo de incontinencia urinaria en adultos mayores varones que asisten al consultorio externo de medicina de un Hospital público de II nivel Ica, enero 2016. Material y métodos: El estudio fue cuantitativo, de tipo descriptivo, y transversal, la muestra estuvo conformada por 40 adultos mayores, de acuerdo al muestreo no probabilístico y por conveniencia, la técnica que se utilizó fue la encuesta. Resultados: En relación a los datos generales, el 100\% de los adultos mayores son de sexo masculino en su totalidad, El $60 \%$ tienen edades de 60 a 70 años, el $38 \%$ tienen entre 71 a 80 años y el $2 \%$ de 81 años a más, en relación al estado de fragilidad de la salud, el $92 \%$ de los adultos mayores presenta riesgo de dependencia, y el $8 \%$ no, el $40 \%$ tiene incontinencia urinaria de urgencia, y el $60 \%$ no presentan incontinencia urinaria. Conclusiones: El 92\% de los adultos mayores presenta riesgo de dependencia y el $40 \%$ tiene incontinencia urinaria de urgencia, por lo que se recomienda un estilo de vida saludable, donde se mantenga un peso adecuado y que realicen controles médicos periódicos, asimismo reforzar los esfuerzos y trabajar arduamente con la Estrategia Sanitaria de Promoción de la Salud y Adulto mayor realizando mediciones periódicas de la dependencia en el adulto mayor detectando a tiempo alguna discapacidad, tratar adecuadamente, y/o recuperar los factores que impulsan o profundizan dicho proceso para evitar la incapacidad total y dependencia.

Palabras clave: Incontinencia urinaria, adulto mayor.

\section{ABSTRACT}

Objetive: To determine the state of health fragility and type of urinary incontinence in older adults who attend the external medical office of a public hospital of II level Ica, January 2016. Materials and Methods: The study was quantitative, descriptive, and cross - sectional, the sample consisted of 40 elderly adults, according to non probabilistic sampling and for convenience, the technique used was the survey. Results: In relation to general data, $100 \%$ of the elderly are male in their entirety, $60 \%$ are 60 to 70 years old, $38 \%$ are between 71 and 80 years old, and $2 \%$ are 81 Years of age, in relation to the fragile state of health, $92 \%$ of older adults are at risk of dependence, and $8 \%$ do not, $40 \%$ have urinary incontinence, and $60 \%$ do not present urinary incontinence. Conclusions: $92 \%$ of older adults are at risk of dependency and $40 \%$ have urgent urinary incontinence, therefore a healthy lifestyle is recommended, where adequate weight is maintained and regular medical checks are performed, as well as Efforts and work hard with the Health Strategy for Health Promotion and the Elderly, making periodic measurements of dependence on the elderly, detecting in time some disability, treating adequately, and / or recovering the factors that drive or deepen this process to avoid Total incapacity and dependence.

Keywords: Urinary incontinence, elderly 


\section{INTRODUCCIÓN}

En la actualidad, existen en el mundo más de 416 millones de adultos mayores. Para el 2025, los mayores de 60 años serán el $12 \%$ de los habitantes del planeta. De este total de adultos mayores, el $72 \%$ vivirá en los países en desarrollo. Se espera que la esperanza de vida al nacimiento en Latinoamérica, se incremente de los 64.1 años que se tuvieron durante 1985, a 71.8 años para el año 2025. A pesar de la diferencia en el proceso evolutivo de la población, el grupo de 60 años y más, ha tenido incrementos importantes con relación a otros grupos de edad.

Se da un fenómeno entre los varones mayores que alcanzan y pasan los 75 años. En el grupo de 75 a 80 años hay un deterioro en la salud muy importante, donde se agregan padecimientos pulmonares, cardiacos, de incontinencia urinaria y depresión. Los que sobreviven esta etapa y pasan los 80 , parece que disminuyen algunos padecimientos comparados con otros grupos de edad.

Según la encuesta SABE, en el caso de los hombres, el exámen de la próstata lo realiza sólo un 25 por ciento. 1

La salud del adulto mayor es materia de gran importancia social, como ocurre en muchas partes del mundo, la población mayor de 65 años reside en el medio urbano y se espera que esa proporción continúe aumentando.2El $12 \%$ tanto de hombres como mujeres en todo el mundo padece incontinencia urinaria y el $70 \%$ al $80 \%$ de las personas que llegan a los 80 años sufren este problema. 3

Según un estudio en España en la actualidad existen aproximadamente 150.000 hombres que padecen incontinencia urinaria (un $7 \%$ del total). Esta cifra alcanza el $20 \%$ cuando se trata de hombres de más de 65 años y el $50 \%$ cuando se trata de hombres de más de 85 años. Ese mismo estudio fija en EEUU en un $17 \%$ de hombres mayores a 60 años que padecen incontinencia urinaria $(3.400 .000$ hombres).4.

En Mendoza, Argentina, un 46\% de las personas tiene incontinencia en su versión leve, un $26 \%$ moderada y un $27 \%$ fuerte, según se desprende de un estudio impulsado por Kimberly-Clark y realizado por la consultora GFK. El 23\% de los afectados siente vergüenza de pedir ayuda y queda así lejos de obtener alguna solución que le permita mejorar su calidad de vida 5

Algunos estudios demuestran que el $30 \%$ de adultos mayores padece de incontinencia urinaria, de aquellos que refieren el síntoma no reciben ningún tipo de evaluación. Los estudios epidemiológicos indican que es un trastorno de alta prevalencia: en promedio varían las series entre un 10 a 34\% en las personas mayores de 65 años de edad que viven en la comunidad y puede llegar a ser un 50 a $60 \%$ en las instituciones geriátricas y hospitales generales. Estos porcentajes son aún mayores entre las mujeres, aumenta el porcentaje a mayor edad (>75 años) y se relaciona estrechamente con aquellos que presentan alteraciones y/o fallas de las funciones cognitivas y funcionales-físicas, estas cifras son subestimadas ya que sólo una pequeña proporción de los pacientes que la padecen solicitan ayuda por considerarla como parte del envejecimiento normal, además hay miedo y sobretodo vergüenza de los propios pacientes de contárselo a sus familiares. 6

En nuestro caso los pacientes vienen acompañados la mayoría de veces por algún familiar, temen caerse, refieren que no escuchan bien y sienten vergüenza porque cuando salen a la calle tienen que buscar baño porque sienten la necesidad urgente de miccionar.

En tal sentido el objetivo de la investigación fue determinar el estado de fragilidad de la salud y tipo de incontinencia urinaria en adultos mayores varones que asisten al consultorio externo de medicina de un Hospital Público de II nivel Ica, enero 2016.

\section{MATERIALES Y MÉTODOS}

Se realizó una investigación cuantitativa, descriptiva y de corte transversal, haciendo uso del muestreo no probabilístico por conveniencia, considerándose como referencia a 40 adultos mayores usuarios del consultorio de medicina, se utilizó el cuestionario de Barber con la finalidad de identificar el estado de fragilidad, se trata de una escala autoadministrada, el cual se utiliza para detectar a la población adulta mayor susceptible con algún nivel de dependencia, pero en el medio urbano. Para validar el contenido y constructo del instrumento se realizó la validación por juicio de expertos, el instrumento consta de 9 ítems con respuestas dicotómicas $\mathrm{Si}$ (1) -No (o). La puntuación fue: No riesgo de dependencia (o pts.), Riesgo de dependencia (1 a más pts.) Para la valoración de incontinencia urinaria se utilizaron 5 preguntas afirmativas las cuales tienen un valor predictivo positivo en el hombre para incontinencia urinaria de urgencia.

El análisis estadístico de los datos se realizó haciendo uso del paquete informático Microsoft Excel y Microsoft Office Word 2007.

\section{RESULTADOS}

Según datos generales el 60\%(24) tienen edades de 60 a 70 años, el $38 \%$ (15) tienen entre 71 a 80 años y el $2 \%$ (1) de 81 años a más. En relación al estado de fragilidad de la salud el $92 \%(37)$ de los adultos mayores presenta riesgo de dependencia, y el $8 \%(3)$ no presenta riesgo de dependencia. Respecto al tipo de incontinencia, un 40\%(16) tiene incontinencia urinaria de urgencia, y el 60\%(24) no presentan incontinencia urinaria, según los resultados del cuestionario de valoración para incontinencia urinaria en varones.

\section{DISCUSIÓN}

Referente a los datos generales de los adultos mayores en estudio se pueden contrastar con Guimarães P.7 en el cual muestra asociación con la edad (1,06 por cada año a más de edad, IC95: 1,02-1,11). La incontinencia urinaria, no se trata simplemente de una cuestión de edad, ya que está directamente relacionada con otras afecciones como el aumento de tamaño de la próstata, cáncer prostático y trastornos neurológicos como esclerosis múltiple, enfermedad de Parkinson y Alzheimer.

TABLA Na 01: Datos generales de los adultos mayores que asisten al consultorio externo de medicina de un Hospital público de II nivel Ica, enero 2016.

\begin{tabular}{|l|c|c|}
\multicolumn{1}{|c|}{ EDAD } & $\mathbf{F r}$ & $\%$ \\
\hline De 60 a 70 años & 24 & $60 \%$ \\
\hline De 71 a 80 años & 15 & $38 \%$ \\
\hline 81 años a más & 1 & $2 \%$ \\
\hline TOTAL & 40 & $100 \%$ \\
\hline
\end{tabular}

TABLA N $\mathrm{N}^{\mathrm{a}}$ 02: Tipo de incontinencia urinaria en adultos mayores que asisten al consultorio externo de medicina de un Hospital público de II nivel Ica, enero 2016.

\begin{tabular}{|l|c|c|}
\hline INCONTINENCIA URINARIA & Fr & $\%$ \\
\hline DE URGENCIA & 16 & $40 \%$ \\
\hline NO PRESENTA & 24 & $60 \%$ \\
\hline TOTAL & 40 & $100 \%$ \\
\hline
\end{tabular}


Esta población se ve especialmente afectada por la incontinencia urinaria, llevándoles a limitaciones importantes e incluso al aislamiento social y familiar

Respecto al estado de fragilidad de la salud el $92 \%$ de los adultos mayores presenta riesgo de dependencia, y el $8 \%$ no presenta riesgo de dependencia según los resultados del Test de Barber para el medio urbano, resultados similares a los obtenidos por Guimarães P.7 se ha calculado, que la sufren el $37,4 \%$ de la población de estudio. Se debe prevenir la presencia de enfermedades crónicas que pueden ser potencialmente invalidantes y que una vez instaladas deterioran fuertemente la calidad de vida de la población mayor, fomentando un estilo de vida saludable para llegar hacia un envejecimiento satisfactorio que les permita vivir con independencia

Respecto al tipo de incontinencia urinaria, nuestros resultados son casi similares con García M.8, donde los incontinentes son el 93,10\%, de la población de estudio y sólo el $6,9 \%$, no presentan incontinencia; y por último tenemos a Siranaula V, Urgilés S, Viteri M., 9 en el cual la prevalencia global de Incontinencia urinaria fue de $42,86 \%$ en el sexo masculino.

La incontinencia urinaria es "una condición mediante la cual se origina la pérdida involuntaria de orina, a través de la uretra, objetivamente demostrable originando problemas sociales e higiénicos para el individuo"; se puede considerar como un factor de riesgo de discapacidad, prevalente en el adulto mayor y se asocia a una importante comorbilidad y a un incremento en la mortalidad. La atención de enfermería, como servicio de primer contacto, se resalta en la participación para realizar un exhaustivo examen clínico que deberá incluir la búsqueda intencionada de este padecimiento y sus implicaciones médicas y sociales

Dorotea Orem10 explica el concepto de autocuidado como una contribución constante del individuo a su propia existencia: "El autocuidado es una actividad aprendida por los individuos, orientada hacia un objetivo. Es una conducta que existe en situaciones concretas de la vida, dirigida por las personas sobre sí mismas, hacia los demás o hacia el entorno, para regular los factores que afectan a su propio desarrollo y funcionamiento en beneficio de su vida, salud o bienestar". Orem, define el objetivo de la enfermería como: "Ayudar al individuo a llevar a cabo y mantener por sí mismo acciones de auto cuidado para conservar la salud y la vida, recuperarse de la enfermedad y/o afrontar las consecuencias de dicha enfermedad". Además que la enfermera puede utilizar cinco métodos de ayuda: actuar compensando déficits, guiar, enseñar, apoyar y proporcionar un entorno para el desarrollo. Supone trabajar con aspectos relacionados con la motivación y cambio de comportamiento, teniendo en cuenta aspectos novedosos a la hora de atender a los individuos (percepción del problema, capacidad de auto cuidado, barreras o factores que lo dificultan, recursos para el auto cuidado, etc.) y hacer de la educación para la salud la herramienta principal de trabajo.

El personal de enfermería debe profundizar en las medidas no farmacológicas, enfocarse en la capacitación de los pacientes para la realización continua y adecuada de las medidas conservadoras, contribuir con el paciente en la selección de productos protectores adecuados (pañales) que ofrezcan seguridad y confianza; en muchos de los casos, convencer al paciente que el uso de protectores como un recurso de fácil alcance, el cual genera confort, previene lesiones e infecciones de piel, mucosas y del tracto genitourinario, protege contra el olor, incluso es conveniente desde el punto de vista económico.

Por todas estas consideraciones, se sugiere generar planes de Intervención, seguimiento y evaluación del mismo, a través de un cuidado transdiciplinario efectivo, que incluya no sólo la recuperación sino también y, muy destacadamente, la promoción de su salud, el crecimiento individual y familiar. Es en este contexto general donde el profesional de enfermería debe entregar su contribución específica complementando a la ciencia del curar.

GRÁFICO Na 01: Estado de fragilidad de la salud en adultos mayores que asisten al consultorio externo de medicina de un Hospital público de II nivel Ica, enero 2016.

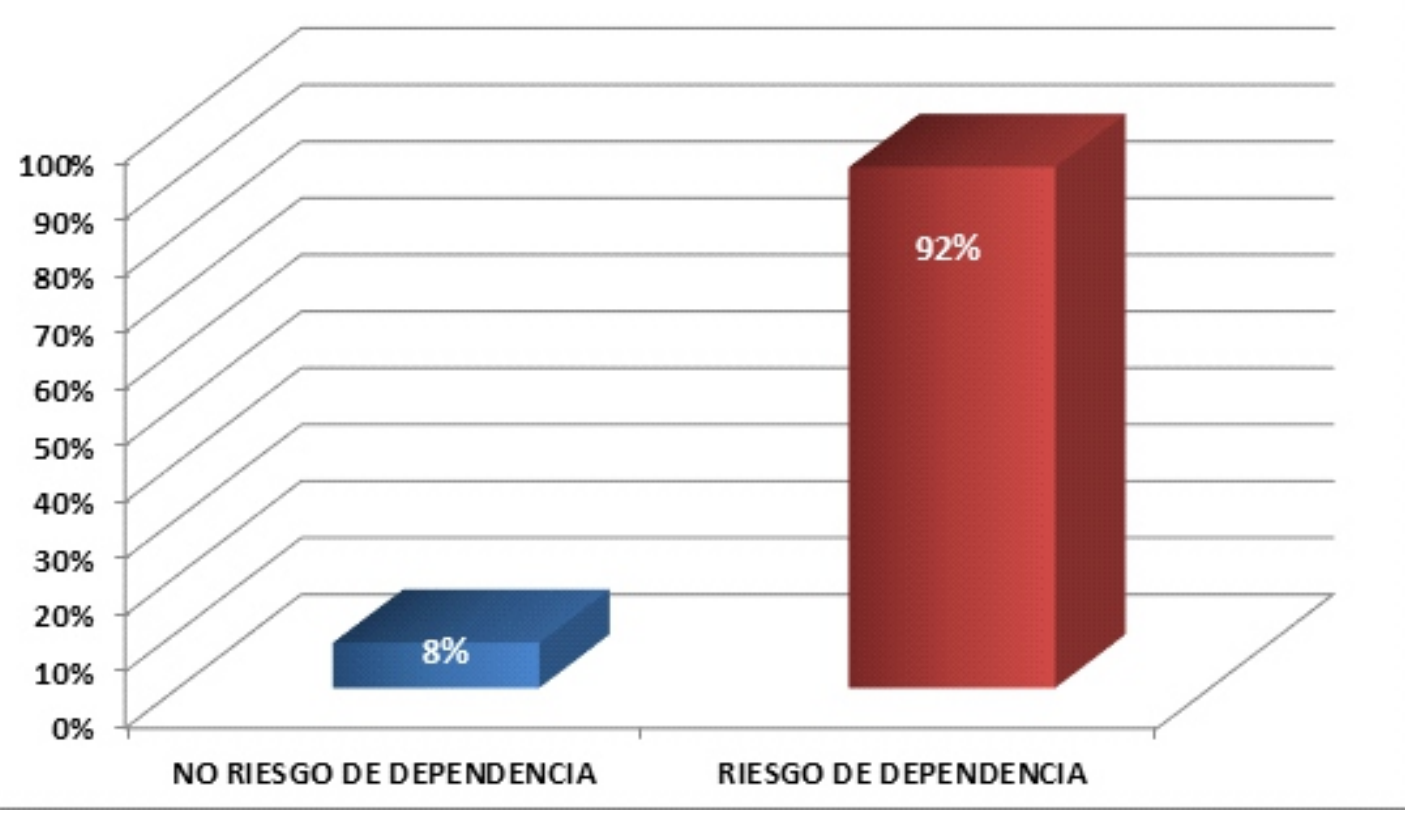




\section{BIBLIOGRAFÍA}

1. Mora J. Programa de Acción: Atención al Envejecimiento Primera edición: 1000 ejemplares. 2006 ISBN 970-721-003-6.

2. Lalive C, Cavalli S, Guilley E. Recorrido de vida y vejez: Sobre la noción de fragilidad. Centro Inter-facultades de Gerontología, Universidad de Ginebra, 2005

3. Costa M. Incontinencia urinaria. Confederación Americana de Urología Hospital Español Infobae Porto Alegre (MN 50407).

4. La incontinencia urinaria masculina: síntomas, tipos, causas y tratamiento Enciclopedia médica Medplus 2 de mayo de 2014.

5. Christian Cobreros. IUBA (Instituto Urológico Buenos Aires).Millón y medio de argentinos sufre incontinencia urinaria Septiembre 2015. www.lanacion.com.ar , Sociedad > Salud

6. Dios JM, Rodríguez M, Martínez Jr, Rodríguez C, Melero M, et al., Prevalencia de la incontinencia urinaria en personas mayores de 64 años en Galicia. Gac Sanit 2003; 17(5):409-11..
7. Guimarães P. Artrosis, estado funcional y fragilidad en una población de personas mayores en Portugal. Estudio de los factores asociados y de la actividad física Oporto, Portugal, [Tesis especialidad]; Universidad autónoma de Madrid. 2012.

8. García M. Tipos de incontinencia urinaria en personas mayores de 65 años institucionalizadas: tratamiento y cuidados en residencias y centros socio-sanitarios públicos de Cataluña, España, 2013.

9. Siranaula Arias V, Urgilés S, Viteri M. Prevalencia y factores de riesgo de la incontinencia urinaria en los adultos mayores que asisten a los servicios sociales del "Instituto Ecuatoriano de Seguridad Social; 2010 $-2011$.

10. Piwonka Ma. Aplicación del modelo de Orem en el cuidado de enfermería a pacientes post operados con desviaciones de salud en el requisito de eliminación Conferencia $51^{\text {a }}$ Congreso Uruguayo de Cirugía. 2000. 